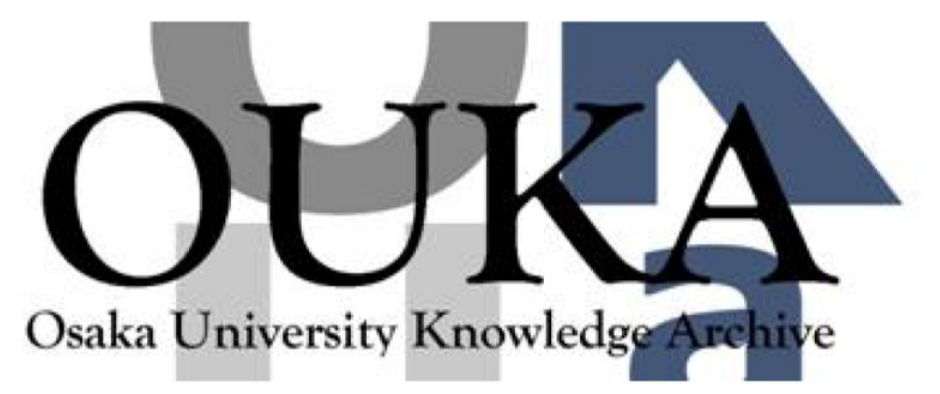

\begin{tabular}{|c|c|}
\hline Title & $\begin{array}{l}\text { The first crystal structure of an alkaline } \\
\text { metal salt of thioglucose : potassium 1-thio- } \\
\beta-D \text {-glucoside monohydrate }\end{array}$ \\
\hline Author (s) & Nobuto, Yoshinari; Naoki, Kitani; Konno, Takumi \\
\hline Citation & $\begin{array}{l}\text { Acta Crystallographica Section C. } 68(12) \\
\text { p. m363-p. } 366 \text { ) }\end{array}$ \\
\hline Issue Date & $2012-12$ \\
\hline oaire:version & VoR \\
\hline URL & https://hdl. handle. net/11094/54383 \\
\hline rights & \\
\hline Note & \\
\hline
\end{tabular}

Osaka University Knowledge Archive : OUKA

https://ir. Library. osaka-u. ac. jp/

Osaka University 


\title{
The first crystal structure of an alkaline metal salt of thioglucose: potassium 1-thio- $\beta$-D-glucoside monohydrate
}

\author{
Nobuto Yoshinari, Naoki Kitani and Takumi Konno
}

Acta Cryst. (2012). C68, m363-m366

\section{W IUCr Journals CRYSTALLOGRAPHY JOURNALS ONLINE \\ Copyright (C) International Union of Crystallography \\ Author(s) of this paper may load this reprint on their own web site or institutional repository provided that this cover page is retained. Republication of this article or its storage in electronic databases other than as specified above is not permitted without prior permission in writing from the IUCr. \\ For further information see http://journals.iucr.org/services/authorrights.html}


Acta Crystallographica Section C

Crystal Structure

Communications

ISSN 0108-2701

\section{The first crystal structure of an alkaline metal salt of thioglucose: potassium 1-thio- $\beta$-D-glucoside monohydrate}

\author{
Nobuto Yoshinari,* Naoki Kitani and Takumi Konno \\ Osaka University, 1-1 Machikaneyama, Toyonaka, Osaka 560-0043, Japan \\ Correspondence e-mail: nobuto@chem.sci.osaka-u.ac.jp
}

Received 10 August 2012

Accepted 15 November 2012

Online 24 November 2012

In the crystal structure of the title hydrated salt, poly $\left[\left(\mu_{2}-\right.\right.$ aqua) $\left(\mu_{4}\right.$-1-sulfido- $\beta$-D-glucoside $)$ potassium], $\left[\mathrm{K}\left(\mathrm{C}_{6} \mathrm{H}_{11} \mathrm{O}_{5} \mathrm{~S}\right)\right.$ $\left.\left(\mathrm{H}_{2} \mathrm{O}\right)\right]_{n}$ or $\mathrm{K}^{+} \cdot \mathrm{C}_{6} \mathrm{H}_{11} \mathrm{O}_{5} \mathrm{~S}^{-} \cdot \mathrm{H}_{2} \mathrm{O}$, each thioglucoside anion coordinates to four $\mathrm{K}^{+}$cations through three of its four hydroxy groups, forming a three-dimensional polymeric structure. The negatively charged thiolate group in each anion does not form an efficient coordination bond with a $\mathrm{K}^{+}$cation, but forms intermolecular hydrogen bonds with four hydroxy groups, which appears to sustain the polymeric structure. The Cremer-Pople parameters for the thioglucoside ligand $(Q=$ $0.575, \theta=8.233^{\circ}$ and $\varphi=353.773^{\circ}$ ) indicate a slight distortion of the pyranose ring.

\section{Comment}

In recent years, thiosugars, especially thiol-containing monosaccharides, have attracted considerable attention; this is because of their unique chemical reactivity due to the presence of the thiol group, as well as the physicochemical and biochemical properties that are inherent in sugars (Witczak \& Culhane, 2005). Among this class of compounds, 1 -thio- $\beta$-Dglucose $\left(\mathrm{H}_{5} \mathrm{tg}\right)$ is one of the simplest thiol-containing hexoses and is commonly used as a raw material for artificial organic thioglycosides. In the field of coordination chemistry, $\mathrm{H}_{5}$ tg has been used as a chiral ligand toward thiophilic metal centres, giving metal complexes with a deprotonated thiolate donor (Okamoto et al., 1994; Leipnitz et al., 2001). Owing to the presence of a sugar backbone in their structures, metal complexes derived from $\mathrm{H}_{5}$ tg often show hydrophilicity and biocompatibility. Hence, $\mathrm{H}_{5}$ tg and/or its metal complexes have been applied not only as antirheumatoid drugs (Shaw, 1999), but also as coating reagents for gold nanoparticles (Watanabe et al., 2010).

While the applications of $\mathrm{H}_{5} \mathrm{tg}$ have developed rapidly, crystallographic information is not extensive. The first structural investigation was made by Waser \& Watson (1963) who elucidated the molecular structure of a $\mathrm{H}_{5} \mathrm{tg}$ residue in Sinigrin [potassium $(E)-1-\{[(2 S, 3 R, 4 S, 5 S, 6 R)-3,4,5$-trihydroxy-6-(hydroxymethyl)oxan-2-yl]sulfanyl but-3-enylideneamino sulfate], which is a kind of thioglycoside extracted from cruciferous plants. After this investigation, a number of S-substituted derivatives of $\mathrm{H}_{5} \mathrm{tg}$ were prepared and structurally characterized (Marsh \& Waser, 1970; Jaki et al., 2002; Kuhn et al., 2004). Nevertheless, the structural determination of $\mathrm{H}_{5}$ tg itself has not yet been reported and, furthermore, only two transition metal complexes derived from $\mathrm{H}_{5} \mathrm{tg}$, namely $\left[\mathrm{Co}\left(\mathrm{H}_{3} \operatorname{tg}-\kappa^{2} S, O\right)\right.$ $\left.(\mathrm{en})_{2}\right]_{\mathrm{NO}_{3}}$ (en is ethylenediamine) [Cambridge Structural Database (CSD) refcode ZIGGOL; Okamoto et al., 1994] and $\left[\mathrm{ReO}(\right.$ tren $\left.)\left(\mathrm{H}_{4} \operatorname{tg}-\kappa S\right)\right]$ [tren is tris(2-aminoethyl)amine; CSD refcode AMONUL; Leipnitz et al., 2001], have been structurally characterized to date. In these complexes, $\mathrm{H}_{5} \mathrm{tg}$ is partially deprotonated and strongly bound to the metal centre through its thiolate donor group.

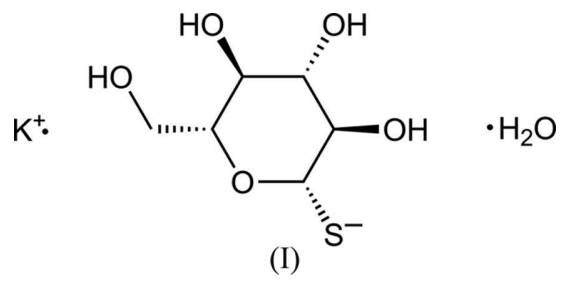

In the course of our study on the preparation of metal complexes derived from $\mathrm{H}_{5} \mathrm{tg}$, we found that the hydrolysis of 2,3,4,6- $O$-acetyl-1-thio- $\beta$-D-glucose with $\mathrm{KOH}$ gave a monohydrate form of potassium 1-thio- $\beta$-D-glucose, viz. $\mathrm{KH}_{4} \mathrm{tg}$. $\mathrm{H}_{2} \mathrm{O}$, (I), which was easily crystallized from water by diffusing acetone. Herein we report the molecular and supramolecular structure of (I). This is the first structural determination of an alkaline metal salt of thioglucose.

Salt (I) consists of one $\mathrm{K}^{+}$cation, one $\mathrm{H}_{4} \mathrm{tg}^{-}$anion and one water molecule, all in general positions in the asymmetric unit (Fig. 1). The absolute configuration of five asymmetric $\mathrm{C}$ atoms $(\mathrm{C} 1, \mathrm{C} 2, \mathrm{C} 3, \mathrm{C} 4$ and $\mathrm{C} 5)$ in the $\mathrm{H}_{4} \mathrm{tg}^{-}$anion were determined to be $S, R, S, S$ and $R$, respectively, based on the Flack (1983) parameter. For the $\mathrm{H}_{4} \mathrm{tg}^{-}$anion, a thiol group is

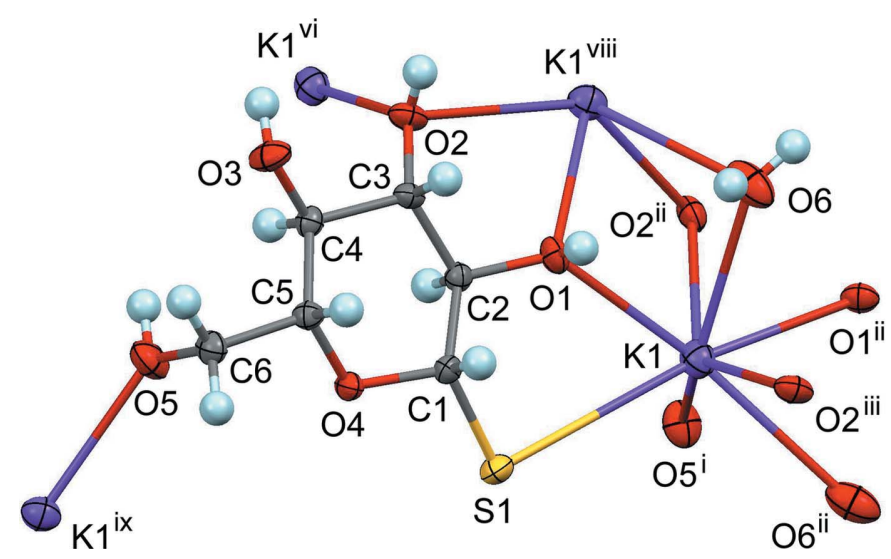

Figure 1

A perspective view of the title compound, showing the atom-numbering scheme. Displacement ellipsoids are drawn at the $70 \%$ probability level. [Symmetry codes: (i) $-x+\frac{3}{2},-y, z+\frac{1}{2}$; (ii) $x-\frac{1}{2},-y+\frac{1}{2},-z+1$; (iii) $x-1$, $y, z$; (vi) $x+1, y, z$; (viii) $x+\frac{1}{2},-y+\frac{1}{2},-z+1$; (ix) $-x+\frac{3}{2},-y, z-\frac{1}{2}$.] 


\section{metal-organic compounds}

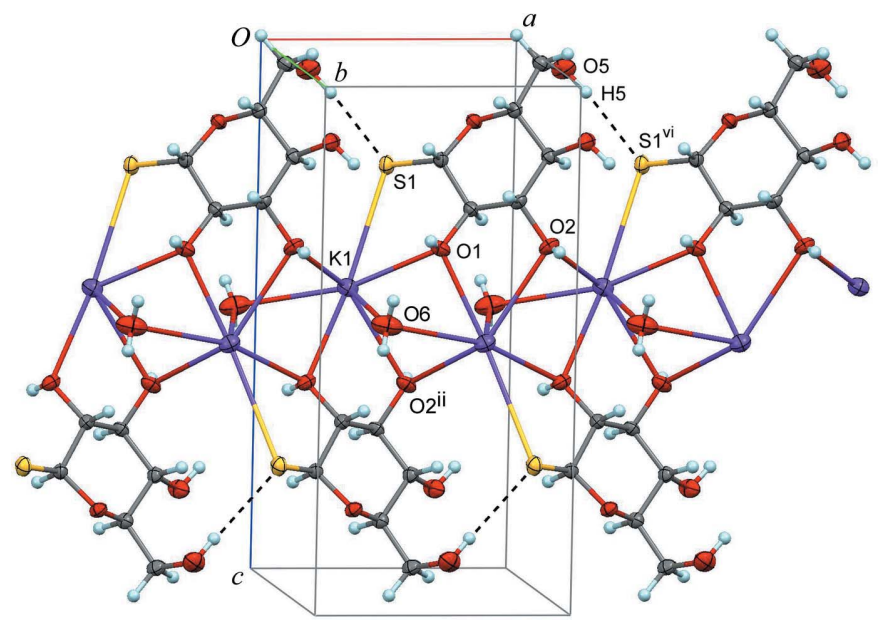

Figure 2

A view of the one-dimensional chain structure along the crystallographic $a$ axis in (I). Dashed lines indicate $\mathrm{O}-\mathrm{H} \cdots \mathrm{S}$ hydrogen bonds. [Symmetry codes: (ii) $x-\frac{1}{2},-y+\frac{1}{2},-z+1$; (vi) $x+1, y, z$.]

deprotonated while four hydroxy groups remain protonated. This is consistent with the higher acidity of a thiol group compared with a hydroxy group. As shown in Fig. 1, a thiolate $\mathrm{S}$ atom and three of four hydroxy $\mathrm{O}$ atoms in each $\mathrm{H}_{4} \mathrm{tg}^{-}$ anion, together with a water $\mathrm{O}$ atom, bind to symmetryrelated $\mathrm{K}^{+}$ions. On the other hand, the $\mathrm{K}^{+}$ion is eight-coordinate, being bound by one thiolate $\mathrm{S}$ atom (S1), four bridging hydroxy $\mathrm{O}$ atoms $\left(\mathrm{O} 1, \mathrm{O} 1^{\mathrm{ii}}, \mathrm{O} 2^{\mathrm{ii}}\right.$ and $\mathrm{O} 2^{\mathrm{iii}}$, with the bridges being to adjacent $\mathrm{K}^{+}$cations) and one monodentate hydroxy $\mathrm{O}$ atom $\left(\mathrm{O}^{\mathrm{i}}\right)$ from four $\mathrm{H}_{4} \mathrm{tg}^{-}$anions, as well as two bridging water $\mathrm{O}$ atoms (O6 and $\mathrm{O}^{\mathrm{ii}}$ ) (symmetry codes as in Fig. 1). It is noteworthy that the $\mathrm{K}-\mathrm{S}$ distance $[\mathrm{K} 1-\mathrm{S} 1=3.5273$ (6) $\AA$; Table 1] is longer than the distances found in general potassium thiolates (3.055-3.216 ̊; Chadwick et al., 1997). This implies that the thiolate group in the $\mathrm{H}_{4} \mathrm{tg}^{-}$anion does not have a high coordination ability to a $\mathrm{K}^{+}$ion. The other bond lengths and angles, including $\mathrm{K}-\mathrm{O}$ bonds [2.7669 (14)3.099 (2) ^], are in the ranges normally observed for related compounds.

In (I), each $\mathrm{K}^{+}$cation is triply bridged to each of two neighbouring $\mathrm{K}^{+}$cations by $\mathrm{O}$ atoms $(\mathrm{O} 1, \mathrm{O} 2, \mathrm{O} 6$ and their symmetry-related counterparts) to give a one-dimensional chain structure along the crystallographic $a$ axis (Fig. 2). This chain structure is supported by $\mathrm{K}-\mathrm{S}$ bonding interactions and intermolecular $\mathrm{O}-\mathrm{H} \cdots \mathrm{S}$ hydrogen bonds $\left[\mathrm{O} 5 \cdots \mathrm{S} 1^{\text {vi. }}\right.$; symmetry code: (vi) $x+1, y, z]$. Moreover, each one-dimensional chain connects with four neighbouring chains through a linkage involving the $\mathrm{K} 1-\mathrm{O}^{\mathrm{i}}$ bond (symmetry code as in Fig. 1), completing a three-dimensional network structure in the crystal packing (Fig. 3). There exist four different $\mathrm{O}-$ $\mathrm{H}$... O hydrogen bonds (Table 2) which also support the threedimensional structure in (I).

As described above, the thiolate group of the $\mathrm{H}_{4} \mathrm{tg}^{-}$anion does not form an efficient coordination bond with a $\mathrm{K}^{+}$cation but forms intermolecular hydrogen bonds with four hydroxy groups in (I). This result obviously shows that the thiolate group in the $\mathrm{H}_{4} \mathrm{tg}^{-}$anion acts as a good hydrogen-bonding acceptor even when an alkaline metal ion exists in its vicinity. Note that the thiolate group in $\left[\mathrm{Co}\left(\mathrm{H}_{3} \operatorname{tg}-\kappa^{2} S, O\right)(\mathrm{en})_{2}\right] \mathrm{NO}_{3}$ forms only one intermolecular $\mathrm{N}-\mathrm{H} \cdots \mathrm{S}$ hydrogen bond $(\mathrm{N} \cdots \mathrm{S}=3.29 \AA)$ and $\left[\mathrm{ReO}(\operatorname{tren})\left(\mathrm{H}_{4} \operatorname{tg}-\kappa S\right)\right]$ does not form any hydrogen bonds involving the $\mathrm{S}$ atom, which is understood by the formation of a strong coordination bond with the transition metal centre.

In order to estimate the distortion of the pyranose ring, the Cremer-Pople theory (Cremer \& Pople, 1975) is useful. The parameters of $(\mathrm{I}),\left[\mathrm{Co}\left(\mathrm{H}_{3} \operatorname{tg}-\kappa^{2} S, O\right)(\mathrm{en})_{2}\right] \mathrm{NO}_{3}$ and $[\mathrm{ReO}($ tren $)$ $\left.\left(\mathrm{H}_{4} \operatorname{tg}-\kappa S\right)\right]$ are as follows: $Q=0.575, \theta=8.233^{\circ}$ and $\varphi=$

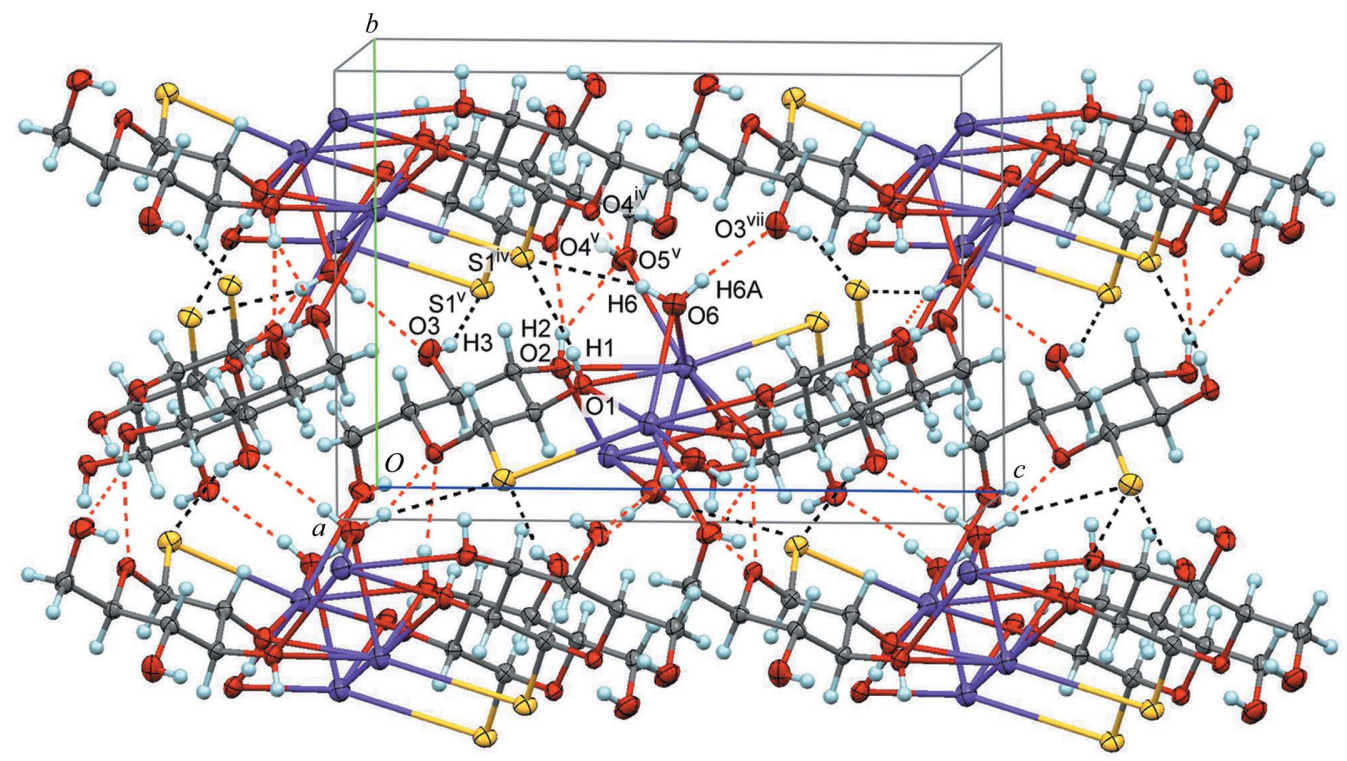

Figure 3

A view of the three-dimensional framework structure in (I). Darker and lighter (black and red in the electronic version of the paper) dashed lines indicate $\mathrm{O}-\mathrm{H} \cdots \mathrm{S}$ and $\mathrm{O}-\mathrm{H} \cdots \mathrm{O}$ hydrogen bonds, respectively. [Symmetry codes: (iv) $-x+1, y+\frac{1}{2},-z+\frac{1}{2} ;(\mathrm{v})-x+2, y+\frac{1}{2},-z+\frac{1}{2} ;$ (vi) $x+1, y, z ;($ vii) $-x+\frac{3}{2},-y+1, z+\frac{1}{2}$.] 
$353.773^{\circ}$ for $(\mathrm{I}) ; Q=0.577, \theta=14.303^{\circ}$ and $\varphi=52.810^{\circ}$ for $\left[\mathrm{Co}\left(\mathrm{H}_{3} \operatorname{tg}-\kappa^{2} S, O\right)(\mathrm{en})_{2}\right] \mathrm{NO}_{3} ; Q=0.555, \theta=3.112^{\circ}$ and $\varphi=$ $313.544^{\circ}$ for $\left[\mathrm{ReO}(\operatorname{tren})\left(\mathrm{H}_{4} \operatorname{tg}-\kappa S\right)\right]$. The $\theta$ value increases for the pyranose ring, which measures the deviation from an ideal chair conformation $\left(\theta=0\right.$ or $\left.180^{\circ}\right)$, in the sequence [ReO$($ tren $\left.)\left(\mathrm{H}_{4} \operatorname{tg}-\kappa S\right)\right]<(\mathrm{I})<\left[\mathrm{Co}\left(\mathrm{H}_{3} \operatorname{tg}-\kappa^{2} S, O\right)(\mathrm{en})_{2}\right] \mathrm{NO}_{3}$. This trend in the ring distortion is explained by the fact that the thioglucoside ligand coordinates to a metal centre in a chelating mode in (I) and $\left[\mathrm{Co}\left(\mathrm{H}_{3} \operatorname{tg}-\kappa^{2} S, O\right)(\text { en })_{2}\right] \mathrm{NO}_{3}$, while the ligand coordinates to a metal centre in a monodentate mode in $\left[\mathrm{ReO}(\operatorname{tren})\left(\mathrm{H}_{4} \operatorname{tg}-\kappa S\right)\right]$. In addition, the ligand coordinates to $\mathrm{a} \mathrm{K}^{+}$centre much more weakly than to a $\mathrm{Co}^{3+}$ centre, which is responsible for the less distorted pyranose ring in (I) compared with that in $\left[\mathrm{Co}\left(\mathrm{H}_{3} \operatorname{tg}-\kappa^{2} S, O\right)(\mathrm{en})_{2}\right] \mathrm{NO}_{3}$.

In summary, this study provides a significant insight into the molecular and supramolecular structures of an alkaline metal salt of 1 -thio- $\beta$-D-glucose for the first time. The crystal structure of (I) is composed of one-dimensional chains sustained by $\mathrm{K}-\mathrm{O}$ coordination bonds, which are further assembled into a three-dimensional structure by forming $\mathrm{K}-\mathrm{O}$ coordination bonds and $\mathrm{O}-\mathrm{H} \cdots \mathrm{O}$ hydrogen bonds. Unlike the known transition metal complexes derived from $\mathrm{H}_{5} \mathrm{tg}$, the thiolate group in $\mathrm{H}_{4} \mathrm{tg}^{-}$does not form an efficient coordination bond with $\mathrm{K}^{+}$in (I) but forms multiple $\mathrm{O}-\mathrm{H} \cdots \mathrm{S}$ hydrogen bonds. This observation demonstrates that the thiolate group in $\mathrm{H}_{4} \mathrm{tg}^{-}$acts as a good hydrogen-bond acceptor, which should contribute to the design and development of new thioglucosebased supramolecular systems.

\section{Experimental}

A sample of 2,3,4,6- $O$-acetyl-1-thio- $\beta$-D-glucose $(4.99 \mathrm{~g}, 0.144 \mathrm{~mol})$ was added to a solution containing $\mathrm{KOH}(2.23 \mathrm{~g}, 0.391 \mathrm{~mol})$ in methanol ( $200 \mathrm{ml}$ ). After stirring for $9 \mathrm{~h}$ under an $\mathrm{N}_{2}$ atmosphere, the resulting white powder was collected by filtration, washed with ethanol and acetone, and dried in vacuo (yield $2.47 \mathrm{~g}, 70 \%$ ). Analysis calculated for $\mathrm{C}_{6} \mathrm{H}_{13} \mathrm{KO}_{6} \mathrm{~S}$ : C 28.56, $\mathrm{H} 5.19 \%$; found: C $28.53, \mathrm{H}$ $4.95 \%$. ${ }^{1} \mathrm{H}$ NMR [400 MHz, $\mathrm{D}_{2} \mathrm{O}$, p.p.m. from sodium 3-(trimethylsilyl)propane-1-sulfonate (DSS)]: $\delta 4.52(1 \mathrm{H}, t, J=9.0 \mathrm{~Hz}), 3.84(1 \mathrm{H}$, $d d, J=12.5,1.5 \mathrm{~Hz}), 3.67-3.64(1 \mathrm{H}, m), 3.42-3.37(3 \mathrm{H}, m), 3.03-2.99$ $(1 \mathrm{H}, m)$. Single crystals of (I) suitable for X-ray analysis were obtained by diffusion of acetone to its aqueous solution.

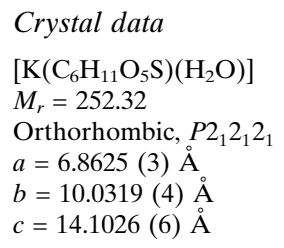

$$
\begin{aligned}
& V=970.88(7) \AA^{3} \\
& Z=4 \\
& \text { Mo } K \alpha \text { radiation } \\
& \mu=0.76 \mathrm{~mm}^{-1} \\
& T=200 \mathrm{~K} \\
& 0.30 \times 0.15 \times 0.15 \mathrm{~mm}
\end{aligned}
$$

\section{Table 1}

\begin{tabular}{|c|c|c|c|c|}
\hline$D-\mathrm{H} \cdots A$ & $D-\mathrm{H}$ & $\mathrm{H} \cdots A$ & $D \cdots A$ & $D-\mathrm{H} \cdots A$ \\
\hline $\mathrm{O} 1-\mathrm{H} 1 \cdots \mathrm{S} 1^{\mathrm{iv}}$ & $0.80(2)$ & $2.49(2)$ & 3.2659 (14) & $164(2)$ \\
\hline $\mathrm{O} 2-\mathrm{H} 2 \cdots \mathrm{O} 4^{v}$ & $0.80(2)$ & $2.27(2)$ & $3.0505(18)$ & $167(3)$ \\
\hline $\mathrm{O} 2-\mathrm{H} 2 \cdots \mathrm{O} 5^{\mathrm{v}}$ & $0.80(2)$ & $2.55(2)$ & $3.026(2)$ & $119(2)$ \\
\hline $\mathrm{O} 3-\mathrm{H} 3 \cdots \mathrm{S} 1^{\mathrm{v}}$ & $0.83(2)$ & $2.41(2)$ & $3.1839(15)$ & $156(2)$ \\
\hline $\mathrm{O} 5-\mathrm{H} 5 \cdots \mathrm{S} 1^{\mathrm{vi}}$ & $0.85(2)$ & $2.61(2)$ & 3.3971 (16) & $154(2)$ \\
\hline $\mathrm{O} 6-\mathrm{H} 6 \cdots \mathrm{O} 4^{\mathrm{iv}}$ & $0.82(2)$ & $2.55(2)$ & $3.163(2)$ & $134(3)$ \\
\hline $\mathrm{O} 6-\mathrm{H} 6 \cdots \mathrm{S} 1^{\mathrm{iv}}$ & $0.82(2)$ & $2.79(2)$ & $3.5839(16)$ & $164(3)$ \\
\hline $\mathrm{O} 6-\mathrm{H} 6 A \cdots \mathrm{O} 3^{\mathrm{vii}}$ & $0.82(2)$ & $2.08(2)$ & $2.892(2)$ & $175(3)$ \\
\hline
\end{tabular}

Selected bond lengths $(\AA)$.

\begin{tabular}{llll}
\hline $\mathrm{K} 1-\mathrm{O} 5^{\mathrm{i}}$ & $2.7669(14)$ & $\mathrm{K} 1-\mathrm{O} 6$ & $2.8774(16)$ \\
$\mathrm{K} 1-\mathrm{O} 1^{\mathrm{ii}}$ & $2.7699(14)$ & $\mathrm{K} 1-\mathrm{O} 2^{\mathrm{ii}}$ & $3.0795(15)$ \\
$\mathrm{K} 1-\mathrm{O} 1$ & $2.7890(14)$ & $\mathrm{K} 1-\mathrm{O} 6^{\mathrm{ii}}$ & $3.099(2)$ \\
$\mathrm{K} 1-\mathrm{O} 2^{\text {iii }}$ & $2.8387(14)$ & $\mathrm{K} 1-\mathrm{S} 1$ & $3.5273(6)$ \\
\hline
\end{tabular}

Symmetry codes: (i) $-x+\frac{3}{2},-y, z+\frac{1}{2}$; (ii) $x-\frac{1}{2},-y+\frac{1}{2},-z+1$; (iii) $x-1, y, z$.
Table 2

Hydrogen-bond geometry $\left(\AA{ }^{\circ}\right)$.

Symmetry codes: (iv) $-x+1, y+\frac{1}{2},-z+\frac{1}{2}$; (v) $-x+2, y+\frac{1}{2},-z+\frac{1}{2}$; (vi) $x+1, y, z$; (vii) $-x+\frac{3}{2},-y+1, z+\frac{1}{2}$.

\section{Data collection}

Rigaku R-AXIS RAPID

diffractometer

Absorption correction: multi-scan (ABSCOR; Rigaku, 1995)

$T_{\min }=0.803, T_{\max }=0.894$

Refinement

$R\left[F^{2}>2 \sigma\left(F^{2}\right)\right]=0.038$

$w R\left(F^{2}\right)=0.078$

$S=1.10$

2209 reflections

145 parameters

6 restraints
9524 measured reflections 2209 independent reflections 2171 reflections with $I>2 \sigma(I)$ $R_{\text {int }}=0.079$
$\mathrm{H}$ atoms treated by a mixture of independent and constrained refinement

$\Delta \rho_{\max }=0.60{\mathrm{e} \AA^{-3}}^{-3}$

$\Delta \rho_{\min }=-0.30 \mathrm{e}^{-3}$

Absolute structure: Flack (1983), 913 Friedel pairs

Flack parameter: -0.01 (4)
$\mathrm{H}$ atoms bound to $\mathrm{C}$ atoms were placed at calculated positions $\left[\mathrm{C}-\mathrm{H}=0.99\left(\mathrm{CH}_{2}\right)\right.$ and $\left.1.00 \AA(\mathrm{CH})\right]$ and refined as riding, with $U_{\text {iso }}(\mathrm{H})=1.2 U_{\text {eq }}(\mathrm{C})$. All $\mathrm{H}$ atoms bound to $\mathrm{O}$ atoms were located in a difference Fourier map and were refined with distance restraints and constrained displacement parameters $[\mathrm{O}-\mathrm{H}=0.84$ (2) $\AA$ and $\left.U_{\text {iso }}(\mathrm{H})=1.2 U_{\text {eq }}(\mathrm{O})\right]$.

Data collection: RAPID-AUTO (Rigaku, 2000); cell refinement: RAPID-AUTO; data reduction: RAPID-AUTO; program(s) used to solve structure: SIR92 (Altomare et al., 1994); program(s) used to refine structure: SHELXL97 (Sheldrick, 2008); molecular graphics: Yadokari-XG 2009 (Kabuto et al., 2009); software used to prepare material for publication: Yadokari-XG 2009.

This work was supported by a Grant-in-Aid for Science Research (grant No. 23350026) from the Ministry of Education, Culture, Sports, Science and Technology of Japan, and by the Kurata memorial Hitachi Science and Technology Foundation.

Supplementary data for this paper are available from the IUCr electronic archives (Reference: EM3054). Services for accessing these data are described at the back of the journal.

\section{References}

Altomare, A., Cascarano, G., Giacovazzo, C., Guagliardi, A., Burla, M. C., Polidori, G. \& Camalli, M. (1994). J. Appl. Cryst. 27, 435.

Chadwick, S., Englich, U. \& Ruhlandt-Senge, K. (1997). Organometallics, 16, 5792-5803.

Cremer, D. \& Pople, J. A. (1975). J. Am. Chem. Soc. 97, 1354-1358.

Flack, H. D. (1983). Acta Cryst. A39, 876-881.

Jaki, B., Sticher, O., Veit, M., Fröhlich, R. \& Pauli, G. F. (2002). J. Nat. Prod. 65 , 517-522. 


\section{metal-organic compounds}

Kabuto, C., Akine, S., Nemoto, T. \& Kwon, E. (2009). Nihon Kessho Gakkaishi, 51, 218-224.

Kuhn, J., Pettersson, E. M., Feld, B. K., Burse, A., Termonia, A., Pasteels, J. M. \& Boland, W. (2004). Proc. Natl Acad. Sci. USA, 101, 13808-13813.

Leipnitz, P., Reck, G., Pietzsch, H.-J. \& Spies, H. (2001). Forschungszent Rossendorf (Ber), 311, 102-103.

Marsh, R. E. \& Waser, J. (1970). Acta Cryst. B26, 1030-1037.

Okamoto, K.-I., Arashi, K., Hidaka, J. \& Konno, T. (1994). Bull. Chem. Soc. Jpn, 67, 2736-3743.
Rigaku (1995). ABSCOR. Rigaku Corporation, Tokyo, Japan.

Rigaku (2000). PROCESS-AUTO. Rigaku Corporation, Tokyo, Japan.

Shaw, C. F. III (1999). Chem. Rev. 99, 2589-2600

Sheldrick, G. M. (2008). Acta Cryst. A64, 112-122.

Waser, J. \& Watson, W. H. (1963). Nature, 198, 1297-1298.

Watanabe, S., Yoshida, K., Shinkawa, K., Kumagawa, D. \& Seguchi, H. (2010) Colloids Surf. A, 81, 570-577.

Witczak, Z. J. \& Culhane, J. M. (2005). Appl. Microbiol. Biotechnol. 69, 237244. 


\title{
supplementary materials
}

Acta Cryst. (2012). C68, m363-m366 [doi:10.1107/S0108270112047014]

\section{The first crystal structure of an alkaline metal salt of thioglucose: potassium 1- thio- $\beta$-D-glucoside monohydrate}

\author{
Nobuto Yoshinari, Naoki Kitani and Takumi Konno
}

Poly $\left[\left(\mu_{2}\right.\right.$-aqua $)\left(\mu_{4}-1\right.$-sulfido- $\beta$-D-glucoside $)$ potassium $]$

Crystal data

$\mathrm{K}^{+} \cdot \mathrm{C}_{6} \mathrm{H}_{11} \mathrm{O}_{5} \mathrm{~S}^{-} \cdot \mathrm{H}_{2} \mathrm{O}$

$M_{r}=252.32$

Orthorhombic, $P 2{ }_{1} 2_{1} 2_{1}$

Hall symbol: P $2 \mathrm{ac} 2 \mathrm{ab}$

$a=6.8625(3) \AA$

$b=10.0319(4) \AA$

$c=14.1026(6) \AA$

$V=970.88(7) \AA^{3}$

$Z=4$

Data collection

Rigaku R-AXIS RAPID

diffractometer

Radiation source: rotating-anode X-ray tube

Detector resolution: 10.000 pixels $\mathrm{mm}^{-1}$

$\omega$ scans

Absorption correction: multi-scan

(ABSCOR; Rigaku, 1995)

$T_{\min }=0.803, T_{\max }=0.894$

Refinement

Refinement on $F^{2}$

Least-squares matrix: full

$R\left[F^{2}>2 \sigma\left(F^{2}\right)\right]=0.038$

$w R\left(F^{2}\right)=0.078$

$S=1.10$

2209 reflections

145 parameters

6 restraints

Primary atom site location: structure-invariant direct methods

Secondary atom site location: difference Fourier map
$F(000)=528$

$D_{\mathrm{x}}=1.726 \mathrm{Mg} \mathrm{m}^{-3}$

Mo $K \alpha$ radiation, $\lambda=0.71075 \AA$

Cell parameters from 9342 reflections

$\theta=3.3-27.5^{\circ}$

$\mu=0.76 \mathrm{~mm}^{-1}$

$T=200 \mathrm{~K}$

Block, colourless

$0.30 \times 0.15 \times 0.15 \mathrm{~mm}$

9524 measured reflections

2209 independent reflections

2171 reflections with $I>2 \sigma(I)$

$R_{\text {int }}=0.079$

$\theta_{\max }=27.5^{\circ}, \theta_{\min }=3.3^{\circ}$

$h=-8 \rightarrow 8$

$k=-12 \rightarrow 12$

$l=-18 \rightarrow 17$

Hydrogen site location: inferred from neighbouring sites

$\mathrm{H}$ atoms treated by a mixture of independent and constrained refinement

$w=1 /\left[\sigma^{2}\left(F_{\mathrm{o}}{ }^{2}\right)+(0.0268 P)^{2}+0.2347 P\right]$

where $P=\left(F_{\mathrm{o}}^{2}+2 F_{\mathrm{c}}{ }^{2}\right) / 3$

$(\Delta / \sigma)_{\max }<0.001$

$\Delta \rho_{\max }=0.60 \mathrm{e} \AA^{-3}$

$\Delta \rho_{\min }=-0.30$ e $\AA^{-3}$

Absolute structure: Flack (1983), 913 Friedel pairs

Flack parameter: -0.01 (4) 


\section{Special details}

Geometry. All e.s.d.'s (except the e.s.d. in the dihedral angle between two 1.s. planes) are estimated using the full covariance matrix. The cell e.s.d.'s are taken into account individually in the estimation of e.s.d.'s in distances, angles and torsion angles; correlations between e.s.d.'s in cell parameters are only used when they are defined by crystal symmetry. An approximate (isotropic) treatment of cell e.s.d.'s is used for estimating e.s.d.'s involving l.s. planes.

Refinement. Refinement of $F^{2}$ against ALL reflections. The weighted $R$-factor $w R$ and goodness of fit $S$ are based on $F^{2}$, conventional $R$-factors $R$ are based on $F$, with $F$ set to zero for negative $F^{2}$. The threshold expression of $F^{2}>\sigma\left(F^{2}\right)$ is used only for calculating $R$-factors(gt) etc. and is not relevant to the choice of reflections for refinement. $R$-factors based on $F^{2}$ are statistically about twice as large as those based on $F$, and $R$ - factors based on ALL data will be even larger.

Fractional atomic coordinates and isotropic or equivalent isotropic displacement parameters $\left(\AA^{2}\right)$

\begin{tabular}{lllll}
\hline & $x$ & $y$ & $z$ & $U_{\text {iso }} / U_{\text {eq }}$ \\
\hline K1 & $0.31561(6)$ & $0.16793(4)$ & $0.45442(3)$ & $0.01885(13)$ \\
S1 & $0.48800(7)$ & $0.05321(4)$ & $0.23337(3)$ & $0.01553(13)$ \\
O1 & $0.6474(2)$ & $0.27794(13)$ & $0.37124(9)$ & $0.0157(3)$ \\
H1 & $0.593(3)$ & $0.3434(19)$ & $0.3516(18)$ & $0.019^{*}$ \\
O2 & $1.0557(2)$ & $0.34495(14)$ & $0.36133(9)$ & $0.0164(3)$ \\
H2 & $1.077(4)$ & $0.4226(17)$ & $0.3659(18)$ & $0.020^{*}$ \\
O3 & $1.1838(2)$ & $0.38611(14)$ & $0.16017(10)$ & $0.0196(3)$ \\
H3 & $1.268(3)$ & $0.409(2)$ & $0.1990(17)$ & $0.023^{*}$ \\
O4 & $0.80599(19)$ & $0.13386(11)$ & $0.14342(9)$ & $0.0130(3)$ \\
O5 & $1.1684(2)$ & $0.07274(13)$ & $0.04992(10)$ & $0.0220(3)$ \\
H5 & $1.251(4)$ & $0.097(2)$ & $0.0918(16)$ & $0.026^{*}$ \\
O6 & $0.4025(3)$ & $0.44051(14)$ & $0.50182(11)$ & $0.0285(4)$ \\
H6 & $0.415(4)$ & $0.481(3)$ & $0.4519(15)$ & $0.034^{*}$ \\
H6A & $0.377(4)$ & $0.493(2)$ & $0.5441(17)$ & $0.034^{*}$ \\
C1 & $0.6660(3)$ & $0.18275(17)$ & $0.21185(12)$ & $0.0117(3)$ \\
H1A & 0.5982 & 0.2622 & 0.1846 & $0.014^{*}$ \\
C2 & $0.7766(3)$ & $0.22580(17)$ & $0.30113(13)$ & $0.0116(4)$ \\
H2A & 0.8406 & 0.1448 & 0.3284 & $0.014^{*}$ \\
C3 & $0.9375(3)$ & $0.32768(17)$ & $0.27842(12)$ & $0.0116(4)$ \\
H3A & 0.8759 & 0.4149 & 0.2616 & $0.014^{*}$ \\
C4 & $1.0637(3)$ & $0.28117(18)$ & $0.19605(13)$ & $0.0121(4)$ \\
H4 & 1.1473 & 0.2051 & 0.2170 & $0.015^{*}$ \\
C5 & $0.9372(3)$ & $0.23761(16)$ & $0.11335(13)$ & $0.0122(4)$ \\
H5A & 0.8579 & 0.3158 & 0.0922 & $0.015^{*}$ \\
C6 & $1.0506(3)$ & $0.18609(18)$ & $0.02932(13)$ & $0.0152(4)$ \\
H6B & 0.9579 & 0.1624 & -0.0217 & $0.018^{*}$ \\
H6C & 1.1353 & 0.2584 & 0.0052 & $0.018^{*}$ \\
\hline
\end{tabular}

Atomic displacement parameters $\left(A^{2}\right)$

\begin{tabular}{lllllll}
\hline & $U^{11}$ & $U^{22}$ & $U^{33}$ & $U^{12}$ & $U^{13}$ & $U^{23}$ \\
\hline $\mathrm{K} 1$ & $0.0211(2)$ & $0.0184(2)$ & $0.0171(2)$ & $-0.00043(16)$ & $0.00256(16)$ & $0.00053(15)$ \\
$\mathrm{S} 1$ & $0.0118(2)$ & $0.0161(2)$ & $0.0188(2)$ & $-0.00296(17)$ & $-0.00001(18)$ & $-0.00087(16)$ \\
O1 & $0.0179(8)$ & $0.0166(6)$ & $0.0125(6)$ & $0.0018(5)$ & $0.0037(6)$ & $-0.0004(5)$ \\
O2 & $0.0194(8)$ & $0.0155(6)$ & $0.0144(6)$ & $-0.0014(5)$ & $-0.0051(5)$ & $-0.0025(5)$ \\
O3 & $0.0193(8)$ & $0.0237(7)$ & $0.0156(7)$ & $-0.0105(6)$ & $0.0001(6)$ & $0.0021(5)$ \\
O4 & $0.0135(7)$ & $0.0134(6)$ & $0.0120(6)$ & $-0.0027(5)$ & $0.0026(5)$ & $-0.0017(4)$
\end{tabular}




\begin{tabular}{lllllll}
\hline O5 & $0.0263(8)$ & $0.0176(7)$ & $0.0220(7)$ & $0.0064(6)$ & $-0.0021(6)$ & $-0.0056(5)$ \\
O6 & $0.0476(11)$ & $0.0181(7)$ & $0.0197(8)$ & $0.0009(7)$ & $0.0056(7)$ & $0.0001(6)$ \\
C1 & $0.0103(9)$ & $0.0125(8)$ & $0.0123(8)$ & $0.0003(7)$ & $-0.0002(6)$ & $-0.0007(6)$ \\
C2 & $0.0109(9)$ & $0.0131(8)$ & $0.0107(8)$ & $0.0006(7)$ & $0.0005(7)$ & $-0.0001(6)$ \\
C3 & $0.0127(9)$ & $0.0110(7)$ & $0.0112(8)$ & $-0.0010(7)$ & $-0.0022(6)$ & $-0.0015(6)$ \\
C4 & $0.0117(10)$ & $0.0123(8)$ & $0.0123(8)$ & $0.0003(6)$ & $0.0008(7)$ & $0.0013(6)$ \\
C5 & $0.0129(10)$ & $0.0117(8)$ & $0.0120(8)$ & $-0.0012(6)$ & $-0.0002(7)$ & $0.0006(6)$ \\
C6 & $0.0164(10)$ & $0.0163(9)$ & $0.0128(8)$ & $-0.0016(7)$ & $0.0029(7)$ & $0.0000(6)$ \\
\hline
\end{tabular}

Geometric parameters $\left(\AA,{ }^{\circ}\right)$

\begin{tabular}{|c|c|c|c|}
\hline $\mathrm{K} 1-\mathrm{O} 5^{\mathrm{i}}$ & $2.7669(14)$ & $\mathrm{O} 4-\mathrm{C} 1$ & $1.447(2)$ \\
\hline $\mathrm{K} 1-\mathrm{O} 1^{\mathrm{ii}}$ & 2.7699 (14) & $\mathrm{O} 5-\mathrm{C} 6$ & $1.425(2)$ \\
\hline $\mathrm{K} 1-\mathrm{O} 1$ & $2.7890(14)$ & $\mathrm{O} 5-\mathrm{K} 1^{\mathrm{vi}}$ & $2.7669(14)$ \\
\hline $\mathrm{K} 1-\mathrm{O} 2^{\mathrm{iii}}$ & $2.8387(14)$ & $\mathrm{O} 5-\mathrm{H} 5$ & $0.852(17)$ \\
\hline $\mathrm{K} 1-\mathrm{O} 6$ & $2.8774(16)$ & $\mathrm{O} 6-\mathrm{K} 1^{\mathrm{iv}}$ & 3.099 (2) \\
\hline $\mathrm{K} 1-\mathrm{O} 2^{\mathrm{ii}}$ & $3.0795(15)$ & $\mathrm{O} 6-\mathrm{H} 6$ & $0.815(18)$ \\
\hline $\mathrm{K} 1-\mathrm{O}^{\mathrm{ii}}$ & 3.099 (2) & $\mathrm{O} 6-\mathrm{H} 6 \mathrm{~A}$ & $0.816(17)$ \\
\hline $\mathrm{K} 1-\mathrm{S} 1$ & $3.5273(6)$ & $\mathrm{C} 1-\mathrm{C} 2$ & $1.532(2)$ \\
\hline $\mathrm{K} 1-\mathrm{H} 1$ & $2.97(2)$ & $\mathrm{C} 1-\mathrm{H} 1 \mathrm{~A}$ & 1.0000 \\
\hline $\mathrm{S} 1-\mathrm{C} 1$ & $1.8092(18)$ & $\mathrm{C} 2-\mathrm{C} 3$ & $1.538(3)$ \\
\hline $\mathrm{O} 1-\mathrm{C} 2$ & 1.427 (2) & $\mathrm{C} 2-\mathrm{H} 2 \mathrm{~A}$ & 1.0000 \\
\hline $\mathrm{O} 1-\mathrm{K} 1^{\mathrm{iv}}$ & $2.7699(14)$ & $\mathrm{C} 3-\mathrm{C} 4$ & $1.522(3)$ \\
\hline $\mathrm{O} 1-\mathrm{H} 1$ & $0.803(17)$ & $\mathrm{C} 3-\mathrm{H} 3 \mathrm{~A}$ & 1.0000 \\
\hline $\mathrm{O} 2-\mathrm{C} 3$ & $1.434(2)$ & $\mathrm{C} 4-\mathrm{C} 5$ & $1.518(3)$ \\
\hline $\mathrm{O} 2-\mathrm{K} 1^{\mathrm{v}}$ & $2.8388(14)$ & $\mathrm{C} 4-\mathrm{H} 4$ & 1.0000 \\
\hline $\mathrm{O} 2-\mathrm{K} 1^{\mathrm{iv}}$ & $3.0795(15)$ & $\mathrm{C} 5-\mathrm{C} 6$ & $1.509(3)$ \\
\hline $\mathrm{O} 2-\mathrm{H} 2$ & $0.795(16)$ & $\mathrm{C} 5-\mathrm{H} 5 \mathrm{~A}$ & 1.0000 \\
\hline $\mathrm{O} 3-\mathrm{C} 4$ & $1.430(2)$ & $\mathrm{C} 6-\mathrm{H} 6 \mathrm{~B}$ & 0.9900 \\
\hline $\mathrm{O} 3-\mathrm{H} 3$ & $0.830(17)$ & $\mathrm{C} 6-\mathrm{H} 6 \mathrm{C}$ & 0.9900 \\
\hline $\mathrm{O} 4-\mathrm{C} 5$ & $1.440(2)$ & & \\
\hline $\mathrm{O} 5^{\mathrm{i}}-\mathrm{K} 1-\mathrm{O} 1^{\mathrm{ii}}$ & $75.85(4)$ & $\mathrm{K} 1^{\mathrm{iv}}-\mathrm{O} 2-\mathrm{H} 2$ & $94.2(19)$ \\
\hline $\mathrm{O} 5^{\mathrm{i}}-\mathrm{K} 1-\mathrm{O} 1$ & $121.18(5)$ & $\mathrm{C} 4-\mathrm{O} 3-\mathrm{H} 3$ & $111.8(19)$ \\
\hline $\mathrm{O} 1 \mathrm{ii}-\mathrm{K} 1-\mathrm{O} 1$ & $129.49(4)$ & $\mathrm{C} 5-\mathrm{O} 4-\mathrm{C} 1$ & $111.49(12)$ \\
\hline $\mathrm{O} 5^{\mathrm{i}}-\mathrm{K} 1-\mathrm{O} 2^{\mathrm{iii}}$ & $142.74(5)$ & $\mathrm{C} 6-\mathrm{O} 5-\mathrm{K} 1^{\mathrm{vi}}$ & $128.28(11)$ \\
\hline $\mathrm{O} 1^{\mathrm{ii}-\mathrm{K}} 1-\mathrm{O} 2^{\mathrm{iii}}$ & $91.49(4)$ & $\mathrm{C} 6-\mathrm{O} 5-\mathrm{H} 5$ & $107.0(18)$ \\
\hline $\mathrm{O} 1-\mathrm{K} 1-\mathrm{O} 2^{\mathrm{iii}}$ & 94.07 (4) & $\mathrm{K} 1^{\mathrm{vi}}-\mathrm{O} 5-\mathrm{H} 5$ & $123.9(18)$ \\
\hline $\mathrm{O} 5^{\mathrm{i}}-\mathrm{K} 1-\mathrm{O} 6$ & $135.07(5)$ & $\mathrm{K} 1-\mathrm{O} 6-\mathrm{K} 1^{\mathrm{iv}}$ & $84.39(4)$ \\
\hline $\mathrm{O} 1 \mathrm{ii}-\mathrm{K} 1-\mathrm{O} 6$ & $72.17(4)$ & $\mathrm{K} 1-\mathrm{O} 6-\mathrm{H} 6$ & $107(2)$ \\
\hline $\mathrm{O} 1-\mathrm{K} 1-\mathrm{O} 6$ & $63.42(5)$ & $\mathrm{K} 1^{\mathrm{iv}}-\mathrm{O} 6-\mathrm{H} 6$ & $104(2)$ \\
\hline $\mathrm{O} 2^{\mathrm{iii}-\mathrm{K} 1-\mathrm{O} 6}$ & $69.09(5)$ & $\mathrm{K} 1-\mathrm{O} 6-\mathrm{H} 6 \mathrm{~A}$ & $138(2)$ \\
\hline 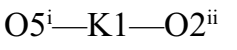 & $62.06(4)$ & $\mathrm{K} 1^{\mathrm{iv}}-\mathrm{O} 6-\mathrm{H} 6 \mathrm{~A}$ & $106(2)$ \\
\hline $\mathrm{O} 1^{\mathrm{ii}-\mathrm{K}} 1-\mathrm{O} 2^{\mathrm{ii}}$ & $58.81(4)$ & $\mathrm{H} 6-\mathrm{O} 6-\mathrm{H} 6 \mathrm{~A}$ & $110(3)$ \\
\hline $\mathrm{O} 1-\mathrm{K} 1-\mathrm{O} 2^{\mathrm{ii}}$ & $86.25(4)$ & $\mathrm{O} 4-\mathrm{C} 1-\mathrm{C} 2$ & $108.34(14)$ \\
\hline 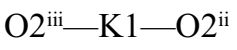 & $138.82(2)$ & $\mathrm{O} 4-\mathrm{C} 1-\mathrm{S} 1$ & $108.46(11)$ \\
\hline $\mathrm{O} 6-\mathrm{K} 1-\mathrm{O} 2^{\mathrm{ii}}$ & $74.52(4)$ & $\mathrm{C} 2-\mathrm{C} 1-\mathrm{S} 1$ & $113.52(12)$ \\
\hline $\mathrm{O} 5^{\mathrm{i}}-\mathrm{K} 1-\mathrm{O}^{\mathrm{ii}}$ & $68.46(5)$ & $\mathrm{O} 4-\mathrm{C} 1-\mathrm{H} 1 \mathrm{~A}$ & 108.8 \\
\hline 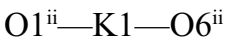 & $60.71(4)$ & $\mathrm{C} 2-\mathrm{C} 1-\mathrm{H} 1 \mathrm{~A}$ & 108.8 \\
\hline $\mathrm{O} 1-\mathrm{K} 1-\mathrm{O} 6^{\mathrm{ii}}$ & $165.85(4)$ & $\mathrm{S} 1-\mathrm{C} 1-\mathrm{H} 1 \mathrm{~A}$ & 108.8 \\
\hline
\end{tabular}




\begin{tabular}{|c|c|c|c|}
\hline 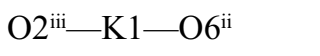 & $74.74(4)$ & $\mathrm{O} 1-\mathrm{C} 2-\mathrm{C} 1$ & $111.40(15)$ \\
\hline $\mathrm{O} 6-\mathrm{K} 1-\mathrm{O} 6^{\mathrm{ii}}$ & $118.48(5)$ & $\mathrm{O} 1-\mathrm{C} 2-\mathrm{C} 3$ & $110.28(14)$ \\
\hline $\mathrm{O} 2^{\mathrm{ii}}-\mathrm{K} 1-\mathrm{O} 6^{\mathrm{ii}}$ & $107.86(4)$ & $\mathrm{C} 1-\mathrm{C} 2-\mathrm{C} 3$ & $111.82(14)$ \\
\hline $\mathrm{O} 5^{\mathrm{i}}-\mathrm{K} 1-\mathrm{S} 1$ & $97.60(3)$ & $\mathrm{O} 1-\mathrm{C} 2-\mathrm{H} 2 \mathrm{~A}$ & 107.7 \\
\hline 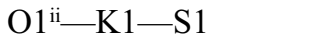 & $171.19(3)$ & $\mathrm{C} 1-\mathrm{C} 2-\mathrm{H} 2 \mathrm{~A}$ & 107.7 \\
\hline $\mathrm{O} 1-\mathrm{K} 1-\mathrm{S} 1$ & $58.91(3)$ & $\mathrm{C} 3-\mathrm{C} 2-\mathrm{H} 2 \mathrm{~A}$ & 107.7 \\
\hline 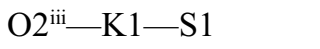 & $90.35(3)$ & $\mathrm{O} 2-\mathrm{C} 3-\mathrm{C} 4$ & $109.74(14)$ \\
\hline $\mathrm{O} 6-\mathrm{K} 1-\mathrm{S} 1$ & $116.48(4)$ & $\mathrm{O} 2-\mathrm{C} 3-\mathrm{C} 2$ & $108.46(14)$ \\
\hline 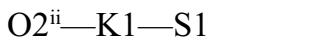 & $123.54(3)$ & $\mathrm{C} 4-\mathrm{C} 3-\mathrm{C} 2$ & $111.31(14)$ \\
\hline $\mathrm{O} 6^{\mathrm{ii}}-\mathrm{K} 1-\mathrm{S} 1$ & $111.61(3)$ & $\mathrm{O} 2-\mathrm{C} 3-\mathrm{H} 3 \mathrm{~A}$ & 109.1 \\
\hline 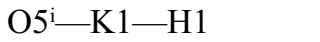 & $136.8(4)$ & $\mathrm{C} 4-\mathrm{C} 3-\mathrm{H} 3 \mathrm{~A}$ & 109.1 \\
\hline $\mathrm{O} 1 \mathrm{ii}-\mathrm{K} 1-\mathrm{H} 1$ & $125.7(5)$ & $\mathrm{C} 2-\mathrm{C} 3-\mathrm{H} 3 \mathrm{~A}$ & 109.1 \\
\hline $\mathrm{O} 1-\mathrm{K} 1-\mathrm{H} 1$ & $15.6(4)$ & $\mathrm{O} 3-\mathrm{C} 4-\mathrm{C} 5$ & $105.65(15)$ \\
\hline $\mathrm{O} 2^{\mathrm{iii}-}-\mathrm{K} 1-\mathrm{H} 1$ & $78.9(4)$ & $\mathrm{O} 3-\mathrm{C} 4-\mathrm{C} 3$ & $111.86(14)$ \\
\hline $\mathrm{O} 6-\mathrm{K} 1-\mathrm{H} 1$ & $54.4(5)$ & $\mathrm{C} 5-\mathrm{C} 4-\mathrm{C} 3$ & $110.45(15)$ \\
\hline 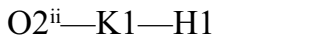 & $95.4(4)$ & $\mathrm{O} 3-\mathrm{C} 4-\mathrm{H} 4$ & 109.6 \\
\hline $\mathrm{O} 6{ }^{\mathrm{ii}}-\mathrm{K} 1-\mathrm{H} 1$ & $153.1(4)$ & $\mathrm{C} 5-\mathrm{C} 4-\mathrm{H} 4$ & 109.6 \\
\hline $\mathrm{S} 1-\mathrm{K} 1-\mathrm{H} 1$ & $63.1(5)$ & $\mathrm{C} 3-\mathrm{C} 4-\mathrm{H} 4$ & 109.6 \\
\hline $\mathrm{C} 1-\mathrm{S} 1-\mathrm{K} 1$ & $98.07(6)$ & $\mathrm{O} 4-\mathrm{C} 5-\mathrm{C} 6$ & $107.83(14)$ \\
\hline $\mathrm{C} 2-\mathrm{O} 1-\mathrm{K} 1^{\mathrm{iv}}$ & $115.34(11)$ & $\mathrm{O} 4-\mathrm{C} 5-\mathrm{C} 4$ & $109.83(15)$ \\
\hline $\mathrm{C} 2-\mathrm{O} 1-\mathrm{K} 1$ & $130.80(11)$ & $\mathrm{C} 6-\mathrm{C} 5-\mathrm{C} 4$ & $114.00(16)$ \\
\hline $\mathrm{K} 1^{\mathrm{iv}}-\mathrm{O} 1-\mathrm{K} 1$ & $92.55(4)$ & $\mathrm{O} 4-\mathrm{C} 5-\mathrm{H} 5 \mathrm{~A}$ & 108.3 \\
\hline $\mathrm{C} 2-\mathrm{O} 1-\mathrm{H} 1$ & $110.3(19)$ & $\mathrm{C} 6-\mathrm{C} 5-\mathrm{H} 5 \mathrm{~A}$ & 108.3 \\
\hline $\mathrm{K} 1^{\mathrm{iv}}-\mathrm{O} 1-\mathrm{H} 1$ & $109.8(18)$ & $\mathrm{C} 4-\mathrm{C} 5-\mathrm{H} 5 \mathrm{~A}$ & 108.3 \\
\hline $\mathrm{K} 1-\mathrm{O} 1-\mathrm{H} 1$ & $95.2(18)$ & $\mathrm{O} 5-\mathrm{C} 6-\mathrm{C} 5$ & $113.95(15)$ \\
\hline $\mathrm{C} 3-\mathrm{O} 2-\mathrm{K} 1^{\mathrm{v}}$ & $131.08(10)$ & $\mathrm{O} 5-\mathrm{C} 6-\mathrm{H} 6 \mathrm{~B}$ & 108.8 \\
\hline $\mathrm{C} 3-\mathrm{O} 2-\mathrm{K} 1^{\mathrm{iv}}$ & $112.36(10)$ & $\mathrm{C} 5-\mathrm{C} 6-\mathrm{H} 6 \mathrm{~B}$ & 108.8 \\
\hline $\mathrm{K} 1^{\mathrm{v}}-\mathrm{O} 2-\mathrm{K} 1^{\mathrm{iv}}$ & 85.39 (4) & $\mathrm{O} 5-\mathrm{C} 6-\mathrm{H} 6 \mathrm{C}$ & 108.8 \\
\hline $\mathrm{C} 3-\mathrm{O} 2-\mathrm{H} 2$ & $106.8(18)$ & $\mathrm{C} 5-\mathrm{C} 6-\mathrm{H} 6 \mathrm{C}$ & 108.8 \\
\hline $\mathrm{K} 1^{\mathrm{v}}-\mathrm{O} 2-\mathrm{H} 2$ & $117.2(19)$ & $\mathrm{H} 6 \mathrm{~B}-\mathrm{C} 6-\mathrm{H} 6 \mathrm{C}$ & 107.7 \\
\hline $\mathrm{O} 5-\mathrm{K} 1-\mathrm{S} 1-\mathrm{C} 1$ & $-132.51(7)$ & $\mathrm{K} 1-\mathrm{S} 1-\mathrm{C} 1-\mathrm{O} 4$ & $159.21(10)$ \\
\hline $\mathrm{O} 1-\mathrm{K} 1-\mathrm{S} 1-\mathrm{C} 1$ & $-10.54(7)$ & $\mathrm{K} 1-\mathrm{S} 1-\mathrm{C} 1-\mathrm{C} 2$ & $38.74(13)$ \\
\hline $\mathrm{O} 22^{\mathrm{iii}}-\mathrm{K} 1-\mathrm{S} 1-\mathrm{C} 1$ & $84.00(7)$ & $\mathrm{K} 1^{\mathrm{iv}}-\mathrm{O} 1-\mathrm{C} 2-\mathrm{C} 1$ & $172.83(10)$ \\
\hline $\mathrm{O} 6-\mathrm{K} 1-\mathrm{S} 1-\mathrm{C} 1$ & $17.31(7)$ & $\mathrm{K} 1-\mathrm{O} 1-\mathrm{C} 2-\mathrm{C} 1$ & $54.55(19)$ \\
\hline 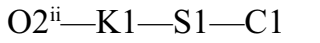 & $-71.11(7)$ & $\mathrm{K} 1^{\mathrm{iv}}-\mathrm{O} 1-\mathrm{C} 2-\mathrm{C} 3$ & $-62.39(15)$ \\
\hline $\mathrm{O} 6{ }^{\mathrm{ii}}-\mathrm{K} 1-\mathrm{S} 1-\mathrm{C} 1$ & $157.70(7)$ & $\mathrm{K} 1-\mathrm{O} 1-\mathrm{C} 2-\mathrm{C} 3$ & $179.33(10)$ \\
\hline $\mathrm{O} 5^{\mathrm{i}}-\mathrm{K} 1-\mathrm{O} 1-\mathrm{C} 2$ & $57.77(15)$ & $\mathrm{O} 4-\mathrm{C} 1-\mathrm{C} 2-\mathrm{O} 1$ & $178.78(13)$ \\
\hline $\mathrm{O} 1 \mathrm{ii}-\mathrm{K} 1-\mathrm{O} 1-\mathrm{C} 2$ & $155.14(13)$ & $\mathrm{S} 1-\mathrm{C} 1-\mathrm{C} 2-\mathrm{O} 1$ & $-60.69(18)$ \\
\hline $\mathrm{O} 2^{\mathrm{iii}-\mathrm{K} 1-\mathrm{O} 1-\mathrm{C} 2}$ & $-109.56(14)$ & $\mathrm{O} 4-\mathrm{C} 1-\mathrm{C} 2-\mathrm{C} 3$ & $54.87(18)$ \\
\hline $\mathrm{O} 6-\mathrm{K} 1-\mathrm{O} 1-\mathrm{C} 2$ & $-173.73(15)$ & $\mathrm{S} 1-\mathrm{C} 1-\mathrm{C} 2-\mathrm{C} 3$ & $175.40(12)$ \\
\hline $\mathrm{O} 22^{\mathrm{ii}}-\mathrm{K} 1-\mathrm{O} 1-\mathrm{C} 2$ & $111.72(14)$ & $\mathrm{K} 1^{\mathrm{v}}-\mathrm{O} 2-\mathrm{C} 3-\mathrm{C} 4$ & $-53.89(19)$ \\
\hline $\mathrm{O} 6{ }^{\mathrm{ii}}-\mathrm{K} 1-\mathrm{O} 1-\mathrm{C} 2$ & $-72.4(2)$ & $\mathrm{K} 1^{\mathrm{iv}}-\mathrm{O} 2-\mathrm{C} 3-\mathrm{C} 4$ & $-157.98(10)$ \\
\hline $\mathrm{S} 1-\mathrm{K} 1-\mathrm{O} 1-\mathrm{C} 2$ & $-21.61(13)$ & $\mathrm{K} 1^{\mathrm{v}}-\mathrm{O} 2-\mathrm{C} 3-\mathrm{C} 2$ & $67.90(18)$ \\
\hline $\mathrm{O} 5^{\mathrm{i}}-\mathrm{K} 1-\mathrm{O} 1-\mathrm{K} 1^{\mathrm{iv}}$ & $-69.41(5)$ & $\mathrm{K} 1^{\mathrm{iv}}-\mathrm{O} 2-\mathrm{C} 3-\mathrm{C} 2$ & $-36.18(15)$ \\
\hline $\mathrm{O} 1^{\mathrm{ii}-}-\mathrm{K} 1-\mathrm{O} 1-\mathrm{K} 1^{\mathrm{iv}}$ & $27.96(6)$ & $\mathrm{O} 1-\mathrm{C} 2-\mathrm{C} 3-\mathrm{O} 2$ & $65.60(18)$ \\
\hline 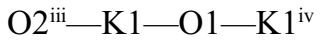 & $123.26(4)$ & $\mathrm{C} 1-\mathrm{C} 2-\mathrm{C} 3-\mathrm{O} 2$ & $-169.86(15)$ \\
\hline $\mathrm{O} 6-\mathrm{K} 1-\mathrm{O} 1-\mathrm{K} 1^{\mathrm{iv}}$ & $59.09(5)$ & $\mathrm{O} 1-\mathrm{C} 2-\mathrm{C} 3-\mathrm{C} 4$ & $-173.58(15)$ \\
\hline 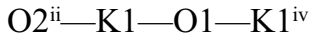 & $-15.46(4)$ & $\mathrm{C} 1-\mathrm{C} 2-\mathrm{C} 3-\mathrm{C} 4$ & $-49.0(2)$ \\
\hline
\end{tabular}




\section{supplementary materials}

\begin{tabular}{|c|c|c|c|}
\hline $\mathrm{O} 6^{\mathrm{ii}}-\mathrm{K} 1-\mathrm{O} 1-\mathrm{K} 1^{\mathrm{iv}}$ & $160.44(15)$ & $\mathrm{O} 2-\mathrm{C} 3-\mathrm{C} 4-\mathrm{O} 3$ & $-73.20(18)$ \\
\hline $\mathrm{S} 1-\mathrm{K} 1-\mathrm{O} 1-\mathrm{K} 1^{\mathrm{iv}}$ & $-148.79(5)$ & $\mathrm{C} 2-\mathrm{C} 3-\mathrm{C} 4-\mathrm{O} 3$ & $166.72(15)$ \\
\hline 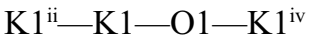 & $87.04(5)$ & $\mathrm{O} 2-\mathrm{C} 3-\mathrm{C} 4-\mathrm{C} 5$ & $169.42(13)$ \\
\hline 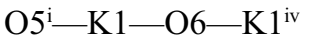 & $58.21(8)$ & $\mathrm{C} 2-\mathrm{C} 3-\mathrm{C} 4-\mathrm{C} 5$ & $49.34(19)$ \\
\hline 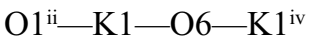 & $104.88(5)$ & $\mathrm{C} 1-\mathrm{O} 4-\mathrm{C} 5-\mathrm{C} 6$ & $-169.13(14)$ \\
\hline $\mathrm{O} 1-\mathrm{K} 1-\mathrm{O} 6-\mathrm{K} 1^{\mathrm{iv}}$ & $-50.34(4)$ & $\mathrm{C} 1-\mathrm{O} 4-\mathrm{C} 5-\mathrm{C} 4$ & $66.10(17)$ \\
\hline 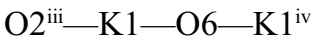 & $-156.37(5)$ & $\mathrm{O} 3-\mathrm{C} 4-\mathrm{C} 5-\mathrm{O} 4$ & $-178.36(13)$ \\
\hline 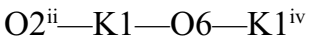 & $43.26(3)$ & $\mathrm{C} 3-\mathrm{C} 4-\mathrm{C} 5-\mathrm{O} 4$ & $-57.22(18)$ \\
\hline $\mathrm{O} 6^{\mathrm{ii}}-\mathrm{K} 1-\mathrm{O} 6-\mathrm{K} 1^{\mathrm{iv}}$ & $145.49(5)$ & $\mathrm{O} 3-\mathrm{C} 4-\mathrm{C} 5-\mathrm{C} 6$ & $60.52(19)$ \\
\hline $\mathrm{S} 1-\mathrm{K} 1-\mathrm{O} 6-\mathrm{K} 1^{\mathrm{iv}}$ & $-76.91(4)$ & $\mathrm{C} 3-\mathrm{C} 4-\mathrm{C} 5-\mathrm{C} 6$ & $-178.34(14)$ \\
\hline 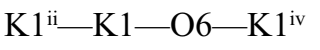 & $150.97(4)$ & $\mathrm{K} 1^{\mathrm{vi}}-\mathrm{O} 5-\mathrm{C} 6-\mathrm{C} 5$ & $128.85(14)$ \\
\hline $\mathrm{C} 5-\mathrm{O} 4-\mathrm{C} 1-\mathrm{C} 2$ & $-64.04(17)$ & $\mathrm{O} 4-\mathrm{C} 5-\mathrm{C} 6-\mathrm{O} 5$ & $-62.2(2)$ \\
\hline $\mathrm{C} 5-\mathrm{O} 4-\mathrm{C} 1-\mathrm{S} 1$ & $172.33(12)$ & $\mathrm{C} 4-\mathrm{C} 5-\mathrm{C} 6-\mathrm{O} 5$ & $60.1(2)$ \\
\hline
\end{tabular}

Symmetry codes: (i) $-x+3 / 2,-y, z+1 / 2$; (ii) $x-1 / 2,-y+1 / 2,-z+1$; (iii) $x-1, y, z$; (iv) $x+1 / 2,-y+1 / 2,-z+1$; (v) $x+1, y, z$; (vi) $-x+3 / 2,-y, z-1 / 2$.

Hydrogen-bond geometry $\left(\AA,{ }^{\circ}\right)$

\begin{tabular}{lllll}
\hline$D-\mathrm{H} \cdots A$ & $D-\mathrm{H}$ & $\mathrm{H} \cdots A$ & $D \cdots A$ & $D-\mathrm{H} \cdots A$ \\
\hline $\mathrm{O} 1-\mathrm{H} 1 \cdots \mathrm{S} 1^{\text {vii }}$ & $0.80(2)$ & $2.49(2)$ & $3.2659(14)$ & $164(2)$ \\
$\mathrm{O} 2-\mathrm{H} 2 \cdots 4^{\text {viii }}$ & $0.80(2)$ & $2.27(2)$ & $3.0505(18)$ & $167(3)$ \\
$\mathrm{O} 2-\mathrm{H} 2 \cdots \mathrm{O} 5^{\text {viii }}$ & $0.80(2)$ & $2.55(2)$ & $3.026(2)$ & $119(2)$ \\
$\mathrm{O} 3-\mathrm{H} 3 \cdots 1^{\text {viii }}$ & $0.83(2)$ & $2.41(2)$ & $3.1839(15)$ & $156(2)$ \\
$\mathrm{O} 5-\mathrm{H} 5 \cdots \mathrm{S} 1^{\text {v }}$ & $0.85(2)$ & $2.61(2)$ & $3.3971(16)$ & $154(2)$ \\
$\mathrm{O} 6-\mathrm{H} 6 \cdots \mathrm{O} 44^{\text {vii }}$ & $0.82(2)$ & $2.55(2)$ & $3.163(2)$ & $134(3)$ \\
$\mathrm{O} 6-\mathrm{H} 6 \cdots \mathrm{S} 1^{\text {vii }}$ & $0.82(2)$ & $2.79(2)$ & $3.5839(16)$ & $164(3)$ \\
$\mathrm{O} 6-\mathrm{H} 6 A \cdots \mathrm{O} 3^{\text {ix }}$ & $0.82(2)$ & $2.08(2)$ & $2.892(2)$ & $175(3)$ \\
\hline
\end{tabular}

Symmetry codes: (v) $x+1, y, z$; (vii) $-x+1, y+1 / 2,-z+1 / 2$; (viii) $-x+2, y+1 / 2,-z+1 / 2$; (ix) $-x+3 / 2,-y+1, z+1 / 2$. 